\title{
¿Educación presencial o a distancia? Reflexiones acerca de su posibilidad y limitaciones.
}

Pág. 186 - 206

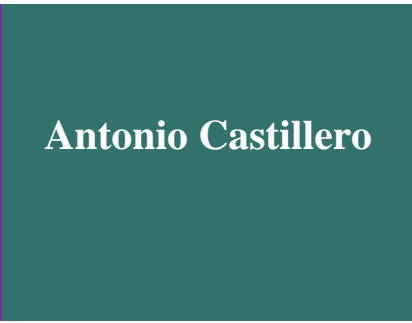

Consultor pedagógico y catedrático universitario castillero.antonio@gmail.com

\section{RESUMEN}

En este escrito se analiza las diferencias entre las modalidades presencial y a distancia y algunos de los aspectos que están en el centro de la problemática de la enseñanza, el aprendizaje y el contenido del currículum. Tal análisis se inscribe en un marco de referencia, que, a manera de antecedentes, dan sentido al texto. Por ejemplo, la evolución de la educación y del lenguaje oral y escrito, la escuela y los sistemas educativos, así como a algunos de los efectos no deseables de la globalización y el desarrollo tecnológico, Se concluye enunciando los aspectos destacados de las relaciones entre esos componentes y la importancia de las competencias para el uso del lenguaje oral y escrito, y de la capacitación de los docentes, entendidos éstas, como acciones que contribuyen a mejorar los resultados de la enseñanza y aprendizaje en cualquiera de las modalidades educativas.

Palabras clave: Contenido del currículum, principios curriculares, educación tradicional, educación a distancia, educación virtual.
Fecha de entrega: noviembre de 2020

Fecha de aceptación: diciembre de 2020 


\title{
Face-to-face or distance education? Thoughts on its possibility and limitations.
}

\begin{abstract}
This writing analyzes the differences between the face-to-face and distance modalities and some of the aspects that are at the center of the problem of teaching, learning and the content of the curriculum. Such analysis is part of a frame of reference, which, as a background, gives meaning to the text. For example, the evolution of education and oral and written language, the school and educational systems, as well as some of the undesirable effects of globalization and technological development. It is concluded by stating the salient aspects of the relationships between these components and the importance of the competences for the use of oral and written language and of the training of teachers. These actions contribute to improving the results of teaching and learning in any of the educational modalities.
\end{abstract}

Keywords: Curriculum content, curricular principles, traditional education, distance education, virtual education. 


\section{INTRODUCCIÓN}

Apenas comenzado el siglo XXI, John Naisbitt (2007, p.27) advertía que "el futuro está incrustado en el presente"; sólo que hay que aprender a leerlo. Años después, la pandemia ocasionada por el coronavirus corrió el velo que cubre las diferencias enormes que hay entre lo que acontece en los centros educativos y su entorno. La realidad mostró diferencias significativas en la expansión del uso de las Tecnologías de la Información y Comunicación (TIC) y la digitalización, que se expresan tanto en la cobertura como en el acceso a estos recursos donde la peor parte la llevan las poblaciones más vulnerables. Los efectos probables de una situación como la que estamos viviendo estaban a la vista; eso es lo que se anunciaba por lo menos hace tres años como resultados de los estudios que tenían como objetivo determinar tanto el acceso como el conocimiento que requiere el uso de la tecnología y la digitalización (Torres, 2020).

El interés por el tema central de estas reflexiones surge por la atención que ha adquirido la modalidad no presencial o a distancia como forma de ofrecer los servicios educativos en los diferentes niveles del sistema; todo esto como consecuencia de la emergencia sanitaria mundial que generó la COVID -19, y las respuestas con las que se busca disminuir sus efectos negativos en todos los países, que según la UNESCO, en mayo de 2020 afectaba cerca de 165 millones de estudiantes en América Latina, y genera una afectación proyectada del 5.5\% en el Producto Interno Bruto (Álvarez et. al., 2020).

Uno de los sectores sociales más afectados por la pandemia del coronavirus es el que se ocupa de la educación institucionalizada. En general, el sistema educativo en todo el mundo, precisó de utilizar las estrategias y los recursos disponibles en un esfuerzo por disminuir los efectos devastadores de la interrupción súbita de la "zona de confort" de la vida cotidiana. En ese proceso, se hicieron más notorias las extremas diferencias económicas y socioculturales que hay entre los distintos sectores de la población estudiantil, la disparidad marcada en las condiciones que permitirían enfrentar con éxito la complejidad del aprendizaje y los procesos educativos, así como las consecuencias negativas de la interrupción indefinida de la presencialidad en los centros educativos. En Panamá se ha procurado enfrentar la transición a 


\section{Antonio Castillero}

un modelo educativo a distancia mediante estrategias y diferentes recursos destinados a lograr la mayor cobertura, igual que se hizo en otros países.

Para mitigar los efectos no deseados, igual que se hizo en otros países, también en Panamá se ha apostado por el uso de diferentes medios de comunicación (radio, TV, teléfono), y la tecnología informática o plataformas educativas diseñadas con el propósito de atender a los estudiantes. Estas actividades son apoyadas mediante diversos recursos y múltiples aplicaciones localizables en diversos motores de búsqueda. En este esquema de funcionamiento surgen interrogantes relacionadas con los modelos educativos, la formación y la capacitación de los docentes; el currículum, las competencias de los estudiantes para usar la tecnología, así como los riesgos inherentes al traslado del modelo presencial al modelo a distancia y su uso en los procesos de enseñanza y aprendizaje. Todo indica que la situación es más complicada de lo que parece, y que, para enfrentarla con mejores posibilidades de éxito, junto con las acciones, también es necesaria la reflexión y el análisis profundo del significado de la educación a distancia.

Este escrito no pretende hacer una interpretación de lo que ocurre en los sistemas educativos en el mundo, o en los establecimientos escolares de los distintos niveles ya sean oficiales o particulares (privados según se les denomine en los distintos países). En su lugar, lo que presento es una reflexión más modesta que procura llamar la atención sobre algunos puntos clave que pueden afectar el logro de los resultados esperados de la educación a distancia en la modalidad virtual; principalmente los relacionados con la comunicación y las competencias el uso del lenguaje entendido como en una totalidad que no se agota en la expresión oral, sino que también abarca al cuerpo. Todo esto dentro de las limitaciones que surgen de la magnitud del problema, según el contexto y formas en que se expresa. 


\section{Educación presencial vs. Educación a distancia: ¿sólo diferencias tecnológicas?}

Desde épocas remotas la educación se ha considerado como un proceso inherente a la sobrevivencia de los seres humanos por eso puede afirmarse que antes, como ahora, el aprendizaje ligado a ella es en esencia estrictamente individual, aunque no tuvo más que un referente que lo explica: la presencia de los otros como requisito necesario en la asimilación de los elementos culturales que constituían la base del sentido de pertenencia e identidad como integrantes de un colectivo.

En ese lejano escenario (con los rasgos que ofrecía la naturaleza y el entorno social) los seres humanos desarrollaron instrumentos que facilitaron la transmisión del rudimentario conocimiento, construido mediante la experiencia real, en el contexto natural, y mediante la participación en la vida comunitaria. Es muy probable que los procesos educativos tuvieran en la imitación su primer recurso de aprendizaje; la comunicación gestual, oral, propiamente dicha llegó después. Entonces todo cambió.

Cabe pensar que los primeros intentos de la comunicación humana fueran gestos y ruidos; la palabra surgió después. También es fácil advertir, que el lenguaje y las habilidades que requiere la observación, considerados como instrumentos de aprendizaje, se convirtieron en recursos insustituibles en la transmisión de la cultura del grupo; principalmente con el propósito de asegurar la supervivencia. Ambos recursos tenían como soporte el cuerpo. De ese modo, cabe suponer que adquirir conocimiento mediante aquellas habilidades solo podían lograrse mediante procesos presenciales guiados; no era posible que existieran procesos de educativos a distancia.

Muchos milenios después, institución educativa de por medio, las TIC se imaginan como herramientas que pueden prescindir del cuerpo y contribuir a una verdadera educación, a sabiendas de que ésta no debe confundirse con el aprendizaje. En relación con este propósito pareciera que la palabra (oral o escrita) y la "presencia" mediante la pantalla de un dispositivo digital son suficientes, sin tomar en cuenta que el cuerpo es un componente esencial de la enseñanza y el aprendizaje. Es el soporte de la racionalidad y de la emocionalidad que están en la base misma de esos componentes de la educación (Aldana, 2019; Heining, 2015). 


\section{Antonio Castillero}

La importancia que se concede a la palabra (oral y escrita) en los procesos educativos institucionalizados tiene una larga historia si se revisa su evolución en los grupos humanos. Frente a las posibilidades que ofrecen las TIC, vale la pena recordar que se puede haber pasado por alto aspectos esenciales de estas habilidades humanas que, según Ong (1987), requieren de un análisis profundo que facilitan la comprensión de su significado y dimensionar su verdadero impacto en la comunicación humana. Según este autor "el cambio de la oralidad a la escritura, y de ahí a la elaboración electrónica, comprometen las estructuras social, económica, política, religiosa y otras" (p.12)

Estas advertencias cobran sentido si se considera que la educación en cualquiera de sus modalidades no puede prescindir del uso de la palabra, ni de las consecuencias de la complejidad de esa evolución y de sus consecuencias. Las evidencias muestran que desde hace alrededor de seis mil años en distintas zonas geográficas, se elaboraron sistemas de representación del lenguaje oral cada uno con características particulares. No cabe duda de que se utilizaron con propósitos de conservación y de transmisión de información, y que las grafías que representan los sonidos y significados de las palabras no son las mismas en todos los grupos.

La tecnología de la escritura cambió el predominio de la palabra y la observación de la realidad; y también las formas de comunicarse y de aprender en cualquier entorno, escolarizado o no, como ocurrió más adelante. Enseñar y aprender, contaban ahora con un tercer instrumento además de la observación y el lenguaje, independiente del gestual como del sonoro sistema oral, externo al cuerpo, y con ventajas. A diferencia de las limitaciones del lenguaje oral, el escrito se puede conservar, transformar, trasladar, reproducir y reutilizar: todo esto, gracias a los diversos soportes que se utilizaron.

Inventada la escritura, la información y el conocimiento se podían difundir ahora por diferentes y variados portadores del texto. En su recuento histórico acerca del libro, Báez (2015) nos describe un conjunto que va desde materiales como el barro, piedra, metales, cuernos, conchas, seda, cuero, o cualquier material que sirviera a esos propósitos, hasta que apareció el papel.

No es necesario explicar por qué los libros se convirtieron en un soporte imprescindible en la realización de las actividades de enseñanza; en su lugar, destacar que este recurso magnificó su 
importancia en la educación gracias a que antes de que finalizara el siglo XV, la invención de la imprenta hizo posible la producción y difusión masiva de textos. El libro adquirió otras características; y la enseñanza, basada casi con exclusividad en el lenguaje oral, encontró apoyo en esta nueva tecnología que permitía realizar todas las acciones mencionadas.

No obstante, su innegable importancia, la palabra impresa no sustituyó la presencia de los maestros, ni del modelo de docencia que predominó en las actividades de la escuela y se mantiene hasta hoy. Por lo que ya se puede percibir, todo parece indicar que no será igual, pues la pandemia del Covid-19 impactará profundamente el predominio del modelo tradicional.

Las instituciones educativas modernas adquirieron sus características esenciales, principalmente a partir de la Revolución industrial y la Revolución Francesa. Así, los propósitos, organización, contenidos, métodos, la evaluación de los aprendizajes, además de las formas en que se producen las interacciones entre sus principales actores, configuraron una imagen de la escuela que aún perdura en gran parte de los sistemas educativos en el mundo. En este panorama, la presencia del maestro o profesor (con su discurso oral), el estudiante y el libro, como recursos esenciales, representaron una trilogía que tiene una larga tradición en la educación escolarizada. Todas las actividades educativas se desarrollaron utilizando métodos y técnicas que encajaban con las formas de entender los propósitos de la educación y la función de las instituciones educativas; los procesos de enseñanza y aprendizaje exigían la presencia de los dos actores principales, el uso de algunos materiales adicionales como ilustraciones en láminas, tiza, tablero y un espacio físico.

Ese modelo de enseñanza basado en la presencialidad, evolucionó lentamente. Y si bien, a lo largo de la historia se advierten algunos cambios internos, mantuvo esas características por lo menos hasta el siglo XIX, cuando surgieron las primeras experiencias de educación a distancia. Esta opción, que permitía estudiar sin aulas y sin maestros o profesores presentes, no tenía regulaciones estrictas de tiempos, o estructuras horarias muy definidas. Uno de los puntos clave de esta modalidad, es su apuesta por el interés y la dedicación de los estudiantes.

Las actividades de educación a distancia crecieron lentamente hasta que, en la segunda mitad del siglo XX adquieren mayor velocidad y relevancia con la creación de la Open University en 


\section{Antonio Castillero}

Inglaterra; pronto le siguieron otras experiencias que impulsaron la difusión y desarrollo de esta forma de enseñar y aprender en diferentes países de Europa y América Latina.

A finales del siglo XIX, García Aretio (1997) hizo un recuento de las modalidades de educación a distancia, cuyo eje central es principalmente una relación no frontal entre docentes y estudiantes. Algunas de estas experiencias, incluían el uso de diferentes medios y herramientas tecnológicas como el correo electrónico, las teleconferencias, el envío de documentos digitalizados mediante Internet y el uso de algunas utilidades de los motores de búsqueda. Estas, y otras aplicaciones, facilitaban la comunicación y el trabajo de los estudiantes en forma individual o en grupo.

Todo eso hasta que, desde la década de los años ochenta del siglo pasado, los avances en el desarrollo de las tecnologías de la información y comunicación llevaron a la invención de otros portadores de texto, sonido e imágenes.

De esta manera, se recuperó aquella característica modelo presencial ya que, mediante nuevos y diversos artilugios, se hizo posible almacenar registros de audio y escuchar las palabras, ver imágenes en movimientos, imágenes virtuales de textos, dibujos, fotografías sin la presencia del emisor. Computadoras, CD-Room, Tablet, USB y teléfonos móviles, permitieron desvincularse de los tradicionales soportes físicos de la escritura, y también se logró almacenar información ilimitadamente en "la nube"; recuperarla y reproducir el conocimiento generado por la humanidad. De esta manera los estudiantes pueden ver y escuchar a sus maestros y profesores en forma sincrónica o asincrónica.

Estos avances dieron origen a distintos tipos de organización de la enseñanza y el aprendizaje: e-learning, b-learning, m-learning; y de paso, a la enseñanza virtual apoyada por las denominadas plataformas educativas con diferentes utilidades, de las cuáles por lo menos 27 son gratuitas. Algunas de éstas son: Moodle, ClassRoom, Chamilo, Mcourser, llias. En Panamá, el Ministerio de Educación elaboró la plataforma Ester dirigida a los estudiantes del último año de la educación media. Que existan estos recursos genera un problema: aprender a usarlos con ese propósito requiere un proceso de capacitación. La opinión generalizada sobre esto es que la educación ha llegado tarde, y el Covid-19 lo puso en evidencia.

Además de los mencionados, se utilizan nuevos programas que facilitan la comunicación 
sincrónica "cara a cara" como Zoom, Meet, Teams, o GoToWebinar, que permiten ampliar los recursos que se utilizan en la educación a distancia. Todos accesibles mediante diferentes dispositivos. Además, mediante internet se puede tener acceso a diferentes motores de búsqueda, disponibles sin costo que sirven de apoyo a las modalidades presenciales o virtuales ofreciendo una aparente fuente inagotable de información de todo tipo, ya sea escrita o visual, contenidas en documentos, libros, informes de investigación, videos, conferencias, presentaciones (PPT) a las que se puede tener acceso sin restricciones mediante la conexión a internet, o más recientemente prescindiendo de dicha conexión.

No obstante, igual que sucede en el espacio exterior donde hay mucha chatarra inservible, también ocurre que "la nube" soporta millones de documentos que contienen información no confiable desde el punto de vista de los criterios que dan fe de su validez científica, tecnológica o humanística.

Por eso, es importante verificar las fuentes de lo que se nos ofrece, pues hay mucha información, cuestionada o superada por los avances y convergencia de los conocimientos que desdibujan los campos disciplinarios, y generan nuevas áreas con contenidos, aplicaciones y problemas con características propias, que no encajan en los planes de estudio de ninguno de los niveles del sistema educativo (Watson, 2017). Que esto ocurra, como se expresó antes, depende de que se reconozca que maestros, profesores y estudiantes, sólo podrán hacer un uso inteligente de esos recursos si cuentan con las competencias y las aptitudes académicas y tecnológicas que les permitan usar los dispositivos que requiere la educación a distancia; especialmente, las que se utilizan en la modalidad virtual. Mucho más ahora que la emergencia sanitaria ha obligado a los sistemas educativos a recurrir a esta forma de entregar los servicios educativos.

A mediados de este año (2020), apenas unos cuantos meses después de que comenzaran a notarse con más fuerza los efectos de la pandemia, desde Guatemala, se hacía un recuento de ellos: la interrupción del aprendizaje, carecer de apoyo por parte de los padres de familia por no contar con la preparación que les permita ayudar en el aprendizaje de sus hijos, desigualdades en el acceso a las herramientas y recursos que ofrece la digitalización; posible abandono o deserción y migración hacia la educación ofrecida por el Estado, impacto negativo en aspectos como la alimentación y la atención a los cuidados de los niños, desconfianza en la calidad de la 


\section{Antonio Castillero}

educación (Azmitia, 2020). Las necesidades detectadas se enfrentaron con los escasos recursos disponibles, y con modelos de atención a los estudiantes que con algunas excepciones reproducían las características de la educación presencial.

Sea o no un panorama como el generado por el Covid-19, trasladar el modelo escolar de la presencialidad a otro de tipo a distancia o "virtual", no resuelve por sí solo los problemas inherentes a la estructura y funcionamiento de los sistemas educativos. Por el contrario, se han generado nuevos problemas y se hacen más notorios otros a los que la educación presencial no ha dado respuestas satisfactorias; por ejemplo, las deficiencias de los estudiantes en la lectura y la escritura como lo muestran diversos estudios realizados en todos los niveles de la educación escolarizada durante varios períodos. Estas dos competencias no se obvian con el modelo a distancia, y mucho menos en su organización virtual.

Las dificultades mencionadas también se presentan en la educación universitaria, si bien con las características propias de cada centro de estudios. Algunos de los rasgos de la problemática en la educación universitaria fueron caracterizados como resultado del Diálogo Virtual realizado entre los Rectores de 9 universidades de América Latina, más una de España y otra de Portugal, convocado por el Banco Interamericano de Desarrollo (BID) y Universia, Universidad de Santander. En este Diálogo entre las universidades, los Rectores reconocían que "como consecuencia de la digitalización forzada, la mayoría de las universidades han tenido que digitalizar el contenido curricular de forma acelerada y precaria, constriñendo la capacidad de planificación y dificultando los canales de comunicación efectiva” (Vicentini, 2020, p.2).

El Diálogo concluía que la pandemia puso de manifiesto problemas relacionados con la infraestructura tecnológica, la evaluación de los aprendizajes, capacitación de los docentes, la brecha digital, la disminución en la inversión dedicada a las actividades y afectaciones en el financiamiento y salud económica de las universidades. Claro que cada caso es diferente y su magnitud depende de muchos factores, lo que no es óbice para afirmar que comparten rasgos con los diferentes niveles del sistema educativo.

Quizás la explicación a varios de los problemas de los escolares en relación con el aprendizaje de esos dos procesos hay que buscarla en lo que la historia afirma acerca de la lengua y el lenguaje; o dicho de otra forma, las tecnologías de la palabra (Ong, 1987). 
Dentro de la modalidad a distancia, los adelantos en el desarrollo de las TIC dieron origen a una nueva forma de ofrecer los servicios educativos a distancia: la denominada "educación virtual". Algunas de sus características difieren sustancialmente de las que tienen lugar en la presencialidad o de otras formas de atender la educación a distancia; entre ellas, la posibilidad de funcionar sin ningún contacto físico, disponibilidad de múltiples recursos documentales y bibliográficos, videos, posibilidad de interacción sincrónica o asincrónica. Esta nueva modalidad contribuyó a generar confianza en las posibilidades que abrían las TIC en el campo de la educación; mismas a las que el sistema educativo accedió muy lentamente, puesto en escena en sus formas extremas por la pandemia generada por el Covid-19, en muchas partes del mundo.

La adopción acelerada de dicha modalidad principalmente fuera de los sistemas establecidos prácticamente revolucionó la forma de ofrecer servicios educativos. Muy pronto las ofertas crecieron y se convirtieron en una opción al modelo escolarizado, sobre todo, por las expectativas que se generaron y las ventajas que ofrecían. Claro que el cumplimiento de todas esas promesas depende de diversos factores que, desde la realidad, permiten advertir el peligro de mayor desigualdad y exclusión de quienes se encuentran en condiciones de mayor vulnerabilidad; no sólo eso, sino también por el riesgo que suponen ofertas sin la calidad esperada.

El uso de la tecnología, y los múltiples y variados recursos con los que cuenta la oferta virtual, se extendió primero en el nivel superior y, ahora, como producto de la pandemia, también en los otros niveles del sistema educativo. Esto significó la expansión de una nueva forma de interacción en la que los sujetos pueden estar separados no solo por la distancia, sino porque pueden no coincidir en el tiempo. De esta manera se pasó de un modelo básicamente presencial a otro que se presentó no sólo como una estrategia dirigida a lograr la mayor cobertura de la población estudiantil, sino como un instrumento de apoyo a la enseñanza y al aprendizaje.

Lo anterior se enmarca en el uso y desarrollo de la tecnología. Esto no indica que hay que confundir las cosas ya que es improcedente llamar educación virtual a cualquier modalidad a distancia; hay diferencias notables en algunas de sus características y posibilidades. Una de ellas es pretender que una sesión por Zoom o Meet, o con cualquier otro recurso semejante sustituye al aula presencial. En este sentido (Shablico, 2012) nos recuerda que: 
El aula es una estructura psicosocial diseñada con propósitos educativos, donde ocurren eventos sociales y psicológicos como resultado de la interacción docente-alumnos, alumno-alumno, en las que el lenguaje y apoyos no verbales tienen especialmente en el docente, intenciones concretas de generar impacto. (p.101).

Esas características no se logran mediante la tecnología disponible, pues una cosa es el aprendizaje y otra la educación. El primero es individual, mientras que la educación no, pues ésta básicamente es un proceso social que adquiere un matiz artificial en su dimensión escolarizada, contrario a lo que ocurre en la sociedad en general. Hay mucha evidencia que permite afirmar que es frecuente que las instituciones educativas se enfoquen más en los contenidos de la propuesta curricular que en su relación con la educación, entendida en su sentido más amplio y en su carácter contextual e histórico.

La modalidad virtual puede acentuar esta distorsión; sus efectos pueden ser opuestos a los esperados, si consideramos el entorno real de los estudiantes.

Aún hay otro problema. Desde este punto de vista, no hay tal cosa como "aprendizaje virtual”. La psicología del aprendizaje, y recientemente los estudios realizados por los neurocientíficos, permiten afirmar que las personas son las que aprenden, que hay mucho más que información e imágenes, y que no hay nada simulado en ello. Se puede aprender utilizando la tecnología disponible, en forma presencial o en la modalidad a distancia; individual o en forma colectiva utilizando hoy las ventajas de la digitalización que permite la construcción de los denominados “Espacios Virtuales de Aprendizaje” (EVA).

No hay que olvidar que el aprendizaje, escolarizado o no, siempre es un proceso que tiene lugar en una persona; un estudiante, que puede aprender en entornos reales o virtuales; en forma individual o colectiva, o mediante organizaciones que alternan la presencialidad y los momentos a distancia con apoyo de los medios tecnologías disponibles (B-learning, E-learning, M-learnig).

Aparte de esas características, también es necesario considerar que, la escuela y la enseñanza tradicional han centrado sus esfuerzos en la información y los procedimientos, contenidos 
curriculares relacionados con el componente cognitivo del aprendizaje; esto, en detrimento de los aspectos socioafectivos que acompañan a ese aprendizaje. El primer conjunto requiere de habilidades cognitivas que permitan al sujeto incorporar información mediante los sentidos, descifrando el significado de diferentes códigos: sonoros, gráficos, kinestésicos, auditivos o lumínicos; no dice nada respecto del otro componente (el socioafectivo), y deja al descubierto un vacío cuyo análisis podría explicar muchos de los problemas de aprendizaje que tienen los estudiantes.

La evidencia científica acerca de la estructura y fisiología del cerebro muestra que el aprendizaje es el resultado de algo más que la cognición, y que su desconocimiento afecta los resultados de ese proceso; en este caso se trata de las emociones. Los estudios realizados en el terreno de la neurociencia se refieren a este otro componente del aprendizaje; particularmente, sus aplicaciones a la educación y a la didáctica.

Hoy existe abundante literatura acerca de esas investigaciones, y del uso de sus resultados cuyo propósito es explicar y orientar el aprendizaje escolarizado. Investigaciones realizadas sobre el funcionamiento del cerebro, cuyo propósito es indagar cómo se generan las emociones y los sentimientos, han fortalecido los argumentos acerca de su impacto en el aprendizaje. Es decir, han contribuido a profundizar el conocimiento acerca de lo que ocurre a partir de las reacciones de nuestra estructura orgánica y a sostener que aprendemos con todo nuestro organismo (Goleman; 2004; 2006; Damasio, 2017; Mora, 2013; Marina y Rimbaud, 2019).

Esos hallazgos también se aplican al aprendizaje escolarizado en cualquiera de sus expresiones y son una advertencia: Si no se presta suficiente atención a dicho componente, y si no se revisan los contenidos y prácticas del modelo, las críticas a las viejas prácticas de la presencialidad se trasladarán a la modalidad a distancia; sea virtual o no.

La educación a distancia en su modalidad virtual descansa en una tecnología más sofisticada hoy que hace cinco años; como se expresó antes; esto es un avance en la forma de ofrecer los servicios educativos y ofrece posibilidades y recursos que enriquecen la enseñanza y el aprendizaje en cualquiera de sus formas.

La historia de los recursos didácticos da cuenta de esa evolución; el uso de diferentes materiales y la combinación de conocimientos provenientes del campo de la óptica en 


\section{Antonio Castillero}

combinación con el recurso de la electricidad, permitieron diseñar y utilizar diferentes aparatos de filmación y proyección que luego permitieron el desarrollo de la cinematografía, la televisión y otros recursos como episcopios, retroproyectores, proyectores de diapositivas.

La última década del siglo XX marcó la popularización de Internet con lo cual se generó una revolución en las comunicaciones; y así, las computadoras pasaron a ocupar el lugar cimero entre los recursos con propósitos diversos en diferentes sectores: industria, economía, medicina, agricultura y también en la educación. Lo demás es historia.

Se puede afirmar que contrario a lo que ocurrió con los sectores que se mencionaron, esta incorporación ha sido lenta en la educación. En la emergencia sanitaria actual, saltan a la vista las desigualdades tradicionales del sistema educativo, y se confirma que éstas no se resuelven desde la tecnología, sino que es necesario tomar decisiones acertadas de carácter político, social, económico, cultural y educativo, pues de ellas dependen la dirección, el contenido, los recursos y las formas que adoptarán las modalidades educativas. (Azmitia, 2020)

Por mucha tecnología que se utilice, lograr una verdadera educación será una tarea con magros resultados si en el centro del proceso no colocamos el sentido de lo que se propone tal como lo plantea Gimeno Sacristán (2013). Es decir que, si se pasa por alto que los sujetos que aprenden no son iguales, y que aprender requiere tener antes el deseo de lograrlo se lograrán algunos frutos positivos (objetivos y subjetivos). Sería muy difícil que estos aprendizajes logren formar una personalidad que integre en forma equilibrada, conocimientos, habilidades cognitivas, destrezas actitudes y valores que trasciendan lo instrumental y utilitario. Es decir, que se expresen en el comportamiento y en las actitudes de los individuos con un elevado sentido de su compromiso personal, profesional y ciudadano respecto de sí mismo, la sociedad y del entorno natural.

Usar la tecnología en la modalidad a distancia no es tan simple como parece, pues no se trata sólo utilizar recursos tecnológicos. Hay múltiples estudios, que destacan diferentes componentes o factores que contribuyen a comprender o elaborar una teoría integrada sobre ese campo de la educación; y sobre todo, revelan la complejidad teórica y práctica de esa modalidad a distancia, tal como la resume García Aretio (2011). 
a) Industrialización (Peters (1971 y 1993); b) Independencia y autonomía; c)Wedemeyer, 1971 y 1981 y Saba, 2003); d) Distancia transaccional(Moore, 1977, 1997); e) Conversación didáctica guiada (Holmberg, 1985); f)Interacción y comunicación (Bååt, 1984; Holmberg, 1995 y Anderson y Elloumi ,2004); g) Comunicación bidireccional (Garrison y Shale, 1987; Garrison y Baynton,1987; Garrison, 1989 y Garrison, 2000); h) Aprendizaje colaborativo (Henri, 1992; Slavin, 1995; Conrad, 2002; Stacey y Rice, 2002); i) Equivalencia (Simonson, 1999). (p.7-8)

En su análisis, García Aretio (2011) deja claro que durante un período de más de treinta años (1971-2002), la educación a distancia como objeto de estudio, se ocupó de aspectos particulares que proponían explicaciones acerca de algunos componentes esa modalidad. Entre los temas reseñados por el autor se encuentran: los procesos de planificación y la comunicación; el estudiante y las características del tipo de aprendizaje o competencias requeridas, la autonomía e independencia de los estudiantes respecto de su aprendizaje; la organización institucional, el objetivo de aprendizaje, el contenido, los resultados, la distancia y la señal de comunicación, los aspectos didácticos y el tipo de experiencias o actividades propuestas a los estudiantes, el aprendizaje colaborativo o la efectividad de los resultados de la educación a distancia.

Como puede apreciarse, son muchos los temas y problemas que se derivan de dichos estudios, y hay gran similitud con los que surgen del análisis de la modalidad presencial. Algunos de ellos se magnifican cuando las interacciones entre los actores sociales de los procesos de enseñanza y aprendizaje se realizan fuera de los espacios naturales de la educación presencial. Esto indicaría la relevancia de considerar que el uso de medios limita en forma considerable las posibilidades comunicativas del cuerpo, uno de los principales recursos que sólo la presencialidad posibilita en toda su magnitud, tal como expresaron Heining (2015) y Aldana (2019).

En la coyuntura actual, cuando la emergencia sanitaria ha exigido un cambio sustancial en las estrategias dirigidas a llevar los servicios educativos a los estudiantes, los elementos que se 


\section{Antonio Castillero}

mencionaron pueden servir de referentes en la reflexión sobre lo que ocurre en la modalidad presencial, analizar los requerimientos de la enseñanza a distancia utilizando o no las TIC, y diseñar acciones de capacitación y actualización de docentes, directivos y supervisores de la educación que contribuyan a disminuir las posibilidades de fallar en el intento. Sobre todo, tener presente que son contextos distintos, y por consiguiente, no cabe "trasplantar" el modelo presencial a la educación a distancia. Esta afirmación está presente desde inicios de la pandemia en diferentes foros, congresos, documentos, libros o artículos de fácil acceso mediante los buscadores de información disponibles en internet: el diálogo didáctico. En el modelo a distancia, su utilización amerita una reflexión acerca de las dicotomías que se advierten en su forma práctica: real-simulado, síncrono-asíncrono, vertical-horizontal; unidireccional-multidireccional, estructurado- flexible (García Aretio, 2011).

Como puede apreciarse, el contexto y las características de la distancia condicionan cada una de las posibilidades de análisis de ese recurso, que de paso plantea el problema de la formación y capacitación de los docentes. En ese sentido, no parece que exista una teoría sobre la educación a distancia que se ocupe de integrar el conocimiento acerca de cómo influyen los diferentes elementos en los resultados esperados, y si esta modalidad puede sustituir por completo lo que ofrece la presencialidad en los procesos educativos escolarizados.

Dos ejemplos describen las discrepancias entre una y otra modalidad,

1. La distancia transaccional. De acuerdo con la teoría de Moore (Citado por García Aretio, 2011), esta no sólo tiene que ver con el espacio físico entre docentes y estudiantes, sino en los aspectos cognitivos que son afectados también por una distancia psicológica que afecta el aprendizaje y que también puede presentarse en las aulas en la modalidad presencial que es más grave cuando los estudiantes se encuentran dispersos en la geografía del país, ya que los ellos pueden desarrollar sentimientos de aislamiento o abandono, como la disminución de su motivación respecto al aprendizaje. Esto es peor si, además, los estudiantes tienen limitada la conectividad, sin olvidar que también los padres de familia comparten ese entorno, y vivirán esa experiencia en su totalidad. 
2. La independencia y autonomía de los estudiantes. Como lo plantea Wedemeyer (Citado por García Aretio, 2011), la distancia entre docentes y estudiantes pone en el centro de la discusión dos aspectos básicos que pueden ser determinantes en los resultados de aprendizaje. La primera pregunta que se deriva de este problema es ¿Los programas de las asignaturas que utilizan los docentes promueven el desarrollo de las aptitudes que requiere el aprendizaje autónomo y la independencia, el pensamiento crítico, la imaginación y la creatividad, competencias que son esenciales si se quiere tener éxito en la modalidad a distancia?

El análisis de los contenidos de los diferentes elementos de los programas de las asignaturas, por lo menos en nuestro país, evidencia que esas competencias no figuran dentro de los enunciados de cada uno de ellos, aunque aparezcan en las descripciones de los objetivos y fundamentos del currículum, así como en los enunciados de las competencias cuando se este enfoque determina qué deben aprender los estudiantes. Revisar lo actuado es una tarea pendiente.

Del contenido del segundo ejemplo se siguen preguntas relacionadas con la capacitación de los docentes $¿$ Tienen la preparación necesaria que facilite orientar a los estudiantes en el desarrollo de aquellas competencias, aún cuando no aparezcan en los programas de asignatura? ¿Su capacitación les permite promover el desarrollo de las habilidades cognitivas que requiere la comprensión, producción, transformación y uso de la información; o las que tienen que ver con los aspectos socioafectivos que le dan contenido a valores y actitudes que determinan la forma en que se expresan las interacciones que ocurren en los procesos de enseñanza y aprendizaje, o en la vida cotidiana? Ambos cuestionamientos generan diferentes problemas. Igual que en el planteamiento anterior, no puede afirmarse que hay satisfacción en ese terreno como parecen mostrar los resultados de algunos de los estudios realizadas por organismos como OCDE (Pisa) o UNESCO (Serce, Terce), entre otros.

Por lo menos en nuestro medio, las actividades de capacitación de docentes están más relacionadas con contenidos, métodos y técnicas orientadas al aprendizaje de información y procedimientos que favorecen la convergencia del pensamiento y de acciones de dudoso aporte 


\section{Antonio Castillero}

al desarrollo de la imaginación y la creatividad. Dicha capacitación dista de estar diseñada con miras a que ellos aprendan a promover las competencias cognitivas que demanda el estudio independiente y la autonomía del pensamiento, en todos los niveles del sistema educativo.

\section{CONCLUSIONES}

De acuerdo con lo planteado se puede sostener que no es válido trasladar una copia de las características del modelo presencial a la educación a distancia, pues difieren en sus posibilidades y formas de desarrollarse, si se toma en cuenta que:

1. La educación a distancia es una opción que puede utilizarse en momentos normales o de emergencia, en forma total o combinada. Utilizar sus ventajas exige que se consideren las características de la población a la que se dirige, pues sus conocimientos y experiencias son las que posibilitan el uso de los medios y recursos tecnológicos impactan los resultados. Estos pueden ser positivos o no, dependiendo de la atención a otros componentes de los procesos de enseñanza y aprendizaje como la capacitación de los docentes y el currículum.

2. De acuerdo con lo anterior, es improcedente esperar que los padres posean la capacitación que les permita ayudar a sus hijos. No cabe esperar que puedan hacer el seguimiento que demandan los aspectos tanto tecnológicos y académicos que implica la enseñanza, pues no hay previsión que les permita avanzar por ese camino. Esto, aparte de reconocer la enorme diferencia entre los hogares, dadas las condiciones socioeconómicas, culturales y educativas de cada uno de ellos, y tener en cuenta que en cada centro educativo se construye una cultura escolar no repetible en la casa.

3. Es imprescindible evitar confundir los entornos virtuales de aprendizaje (EVA) con el mero uso de recursos tecnológicos, ya que la comunicación mediante la radio, el teléfono o la televisión, no tiene las mismas características de un aula virtual, como la sincronía, envío de documentos o almacenaje de éstos mediante una computadora, o la búsqueda inmediata de información o redactar y trabajar con otros apoyados con aplicaciones especialmente diseñada con ese propósito. 
4. Tener mejores posibilidades de éxito, depende de que los docentes tengan experticia en el uso de diferentes plataformas educativas que estén disponibles gratis o a bajo costo. Los docentes deben estar formados y tener la capacitación que facilite la programación de sus clases, atendiendo a las exigencias de un modelo a distancia, incluida la modalidad virtual, que no son una copia del modelo presencial. Por eso, las clases magistrales no pueden ser el método por excelencia si se quiere transmitir información, pues los motores de búsqueda cumplen esa función.

5. Los estudiantes no solo deben tener acceso, sino también los conocimientos y habilidades que facilitan el uso de esas plataformas. Usar un dispositivo con propósitos de aprendizaje sistematizado como el de la escuela, exige competencias diferentes de las de uso corriente de esas tecnologías.

6. No hay que dar por hecho que los estudiantes poseen las competencias que requieren la lectura y la escritura de modo que puedan aprovechar las referencias o desarrollar las actividades que exijan ambos conocimientos. Esto es más grave cuando se trata de comunidades cuya lengua materna no es la que corrientemente utiliza la mayoría de la población.

7. La casa no es la escuela; tampoco las habitaciones son las aulas, de ahí que los docentes deber tener presente que ese entorno tiene pocas posibilidades de ofrecer las mejores condiciones a los estudiantes en su proceso de aprendizaje.

8. Apoyarse en las TIC y todas sus ventajas hace posible atender las necesidades educativas de los estudiantes en todos los niveles del sistema educativo. No implica sólo hacer adecuaciones y superar algunas de las críticas que se hacen a la educación presencial y capacitar a los docentes en el uso de esos recursos. También es preciso adoptar una posición crítica y cautelosa que dirija al análisis a las secuelas encubiertas de la digitalización, bien identificadas por Loveless y Williamson (2017) quienes advierten que los centros educativos han contribuido a formar nuevas "identidades digitales de aprendizaje" alineadas con determinadas formas de percibir y mantener una cierta visión del futuro; identidades caracterizadas por la interactividad, la conexión y la construcción de modelos, programación o simulaciones enmarcados en los beneficios personales que pueden obtenerse (p.31). 


\section{Antonio Castillero}

Una observación final. Lo que enfrentamos hoy puede ser la consecuencia de no haber aprendido a leer las señales del futuro, como lo expresó Naisbitt (2007); sin embargo, una carrera apresurada está muy lejos de alcanzar ese objetivo, pues las promesas de la tecnología que se utilizan en la educación a distancia en todas sus formas, distan mucho de estar al alcance de todos y de resolver la complejidad de los procesos educativos, tal como nos recuerda Rosa María Torres (2020). Por eso, es necesario diseñar estrategias fundamentadas en el conocimiento y respaldadas por decisiones de políticas educativas enfocadas en la superación de las enormes brechas que hay entre los distintos sectores de la sociedad; no exagerar las expectativas, pero si aprovechar las ventajas de ese producto del intelecto humano.

Nada volverá a ser lo mismo que antes de la pandemia; hay que proponerse que no sea peor.

\section{REFERENCIAS}

Aldana, H. (2019). Enseñar y aprender de los pies a la cabeza- TEDxPuraVidaED. Consultado el 21 de junio de 2019. https://www.youtube.com/watch?v=hCnkIMK4Fvc.

Álvarez Marinelli, Horacio: Arias Ortíz, Elena; Bergamachi, Andrea; López Sánchez, Angela; Noli, Alessandra; Ortíz Guerrero, Marcela; Pérez Alfaro, Marcelo; Rieble-Aurborg. Sabine; Rivera, María Camila; Scanone, Rodolfo; Vázquez, Madiey; Viteri. Adriana (2020). La educación en tiempos del coronavirus: Los sistemas educativos en América Latina y el Caribe ante el COVID-19. Banco Interamericano de Desarrollo. http;//publicartion.iadb.org//es/la-educación-en-tiempos-del-coronavirus-los sistemaseducativos-de-américa-latina-y-el-caribe -ante-covid-19.

Azmitia, O. (2020). Repensando una educación desde la Crisis. (Versión Preliminar). Primera edición. Editor Oscar G. Azmitia. En https://www.uols.org/wpcontent/uploads/2020/06/2020-06-29-Repensando-la-educaci\%C3\%B3n-desde-lacrisis.pdf/.

García Aretio, L. (2011). Perspectivas teóricas de la educación a distancia y virtual. Revista española de pedagogía, 249. https://revistadepedagogia.org/ebooks/. 
Gimeno Sacristán, J. (2013). En busca del sentido de la educación. Primera edición. Madrid: Ediciones Morata, S.L.

Heining, P. (2015). Tres competencias para el futuro. [Imagen]. [Consultado el 5 de junio de 2020]. https://www.youtube.com/watch?v-cQVg1LfmGhE/.

Loveless, A., y Williamson, B. (2017). Nuevas Identidades de Aprendizaje en la Era Digital. Primera edición. Madrid: Narcea Ediciones.

Marina, J., y Rimbaud, J. (2019). Biografía de la Humanidad (2a ed.). Ediciones Culturales Paidós. S.A. de C.V.

Moore, M.G. (1977). On a theory of independent study, (Hagen: Fernuniversitat, ZIFF).

Mora T., F. (2013). Sólo se puede aprender lo que se ama. Madrid- Alianza Editorial.

Naisbitt, J. (2007). 11 Mentalidades para prever el futuro. (1a ed., p. 25). Grupo Editorial Norma.

Ong, W. (1987). Oralidad y escritura: Tecnologías de la palabra. Primera edición. México: Fondo de Cultura Económica (FCE).

Shablico, S. (2012). La Comunicación no verbal en el aula. Un análisis en la enseñanza disciplinar. Primera edición. [Ebook]. Montevideo: Cuadernos de Investigación Educativa, Universidad ORT, Uruguay. En, https://revistas.ort.edu.uy/cuadernos-deinvestigacion-educativa/article/view/2710/.

Torres, R. (2020). ¿Está el mundo preparado para la educación virtual? [Blog]. En https://otra-educacion.blogspot.com/2020/04/2020-esta-el-mundo-preparado-paraglobalizar-la-educacion-virtual.html.

Segunda Reunión del Diálogo Virtual con Rectores de Universidades Líderes de América Latina. Banco Interamericano de Desarrollo, Universia, Universidad de Santander. https://publications.iadb.org/publications/spanish/document/La-educacion-superior-entiempos-de-COVID-19-Aportes-de-la-Segunda-Reunion-del-Di\%C3\%A1logo-Virtualcon-Rectores-de-Universidades-Lideres-de-America-Latina.pdf/.

Watson, P. (2017). Convergencias (1a ed.). Barcelona. Editorial Planeta. 